International Journal of Pure and Applied Mathematics

Volume 95 No. 4 2014, 503-514

ISSN: 1311-8080 (printed version); ISSN: 1314-3395 (on-line version)

url: http://www.ijpam.eu

doi: http://dx.doi.org/10.12732/ijpam.v95i4.2

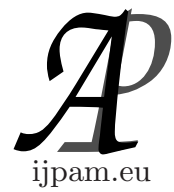

\title{
DELAY-DEPENDENT DECENTRALIZED EXPONENTIAL STABILITY OF NONLINEAR LARGE-SCALE SYSTEMS WITH NON-DIFFERENTIABLE AND INTERVAL TIME-VARYING DELAY IN INTERCONNECTION
}

\author{
Grienggrai Rajchakit \\ Department of Mathematics \\ Maejo University \\ Chiangmai, 50290, THAILAND
}

\begin{abstract}
This paper addresses decentralized exponential stability problem for a class of nonlinear large-scale systems with time-varying delay in interconnection is considered. The time delay is any continuous function belonging to a given interval, but not necessary to be differentiable. By constructing a suitable augmented Lyapunov-Krasovskii functional combined with LeibnizNewton's formula, new delay-dependent sufficient conditions for the existence of decentralized exponential stability is established in terms of LMIs.
\end{abstract}

AMS Subject Classification: 93C30, 93D20, 37C75, 34G20, 93A15

Key Words: decentralized exponential stability, nonlinear large-scale systems, interval time-varying delay, Lyapunov function, linear matrix inequalities

\section{Introduction}

In view of reliability and practical implementation, the decentralized stabilization of large scale interconnected systems becomes a very important problem 
and has been studied extensively for more than two decades. However, the problem of decentralized stabilization of large scale systems with delays has generally been overlooked. The majority of the works on decentralized stabilization are based on interconnection pattens with no delays. In fact, this is generally not the case for many physical systems due to transport and computation time. Large scale interconnected systems can be found in such diverse fields as electrical power systems, space structures, manufacturing process, transportation, and communication.An important motivation for the design of decentralized schemes is that the information exchange between subsystems of a large-scale system is not needed;thus, the individual subsystems controllers are simple and use only locally available information. To the best of our knowledge, interval time-varying delay and exponential stability of nonlinear large-scale systems with time-varying delays interacted between subsystems, non-differentiable time-varying delays have not been fully studied yet (see, e.g., [1-31] and the references therein), which are important in both theories and applications. This motivates our research. In fact, this problem is difficult to solve; particularly, when the time-varying delays are interval, non-differentiable and the output is subjected to such time-varying delay functions.

The objective of this paper is to extend the previous works to nonlinear large-scale systems with time-varying delays interacted between subsystems and develop a new and simple approach for the design of delay-dependent decentralized exponential stability of nonlinear large-scale systems with nondifferentiable and interval time-varying delay in interconnection.

The paper is organized as follows. Section 2 presents definitions and some well-known technical propositions needed for the proof of the main results. Main result for decentralized exponential stability of nonlinear large-scale systems is presented in Section 3.

\section{Preliminaries}

The following noted in this paper. $R^{+}$denotes the set of all real non-negative numbers; $R^{n}$ denotes the $n$-dimensional space with the scalar product $\langle.,$. and the vector norm $\|$. $\| ; M^{n \times r}$ denotes the space of all matrices of $(n \times$ $r$ )-dimensions; $A^{T}$ denotes the transpose of matrix $A ; A$ is symmetric if $A=$ $A^{T} ; I$ denotes the identity matrix; $\lambda(A)$ denotes the set of all eigenvalues of $A ; \lambda_{\min / \max }(A)=\min / \max \{\operatorname{Re} \lambda ; \lambda \in \lambda(A)\} ; C^{1}\left([a, b], R^{n}\right)$ denotes the set of all $R^{n}$-valued differentiable functions on $[a, b] ; L_{2}\left([0, \infty], R^{r}\right)$ stands for the set of all square-integrable $R^{r}$-valued functions on $[0, \infty] . x_{t}:=\{x(t+s)$ : 
$s \in[-h, 0]\},\left\|x_{t}\right\|=\sup _{s \in[-h, 0]}\|x(t+s)\| ; C\left([0, t], R^{n}\right)$ denotes the set of all $R^{n}$-valued continuous functions on $[0, t]$; Matrix $A$ is called semi-positive definite $(A \geq 0)$ if $\langle A x, x\rangle \geq 0$, for all $x \in R^{n} ; A$ is positive definite $(A>0)$ if $\langle A x, x\rangle>0$ for all $x \neq 0 ; A>B$ means $A-B>0$. * denotes the symmetric term in a matrix.

Consider a class of nonlinear large-scale systems with interval time-varying delays composed of $N$ interconnected subsystems $i=\overline{1, N}$ of the form

$$
\begin{gathered}
\dot{x}_{i}(t)=A_{i} x_{i}(t)+\sum_{j \neq i, j=1}^{N} D_{i j} x_{j}(t)+\sum_{j \neq i, j=1}^{N} f_{i}\left(t, x_{i}(t), x_{j}\left(t-h_{i j}(t)\right), \quad t \in R^{+},\right. \\
x_{i}(t)=\varphi_{i}(t), \quad \forall t \in\left[-h_{2}, 0\right],
\end{gathered}
$$

where $x^{T}(t)=\left[x_{1}^{T}(t), \ldots, x_{N}^{T}(t)\right], x_{i}(t) \in R^{n_{i}}$, is the state vector, the systems matrices $A_{i}, D_{i j}$ are of appropriate dimensions.

The nonlinear perturbations $f_{i}\left(t, x_{i}(t), x_{j}\left(t-h_{i j}(t)\right)\right.$ satisfies the following condition

$$
\begin{aligned}
& \sum_{j \neq i, j=1}^{N} f_{i}\left(t, x_{i}(t), x_{j}\left(t-h_{i j}(t)\right) \leq \beta_{i 1} x_{i}(t)+\sum_{j \neq i, j=1}^{N} \beta_{i 2} x_{j}\left(t-h_{i j}(t),\right.\right. \\
& \sum_{j \neq i, j=1}^{N} f_{i}^{T}\left(t, x_{i}(t), x_{j}\left(t-h_{i j}(t)\right) f_{i}\left(t, x_{i}(t), x_{j}\left(t-h_{i j}(t)\right)\right.\right. \\
& \leq \beta_{i 1}^{2} x_{i}^{T}(t) x_{i}(t)+\sum_{j \neq i, j=1}^{N} \beta_{i 2}^{2} x_{j}^{T}\left(t-h_{i j}(t) x_{j}\left(t-h_{i j}(t),\right.\right.
\end{aligned}
$$

where $\beta_{i 1}, \beta_{i 2}, i=\overline{1, N}$ are positive constants. Let us denote that

$$
f_{i}=f_{i}\left(t, x_{i}(t), x_{j}\left(t-h_{i j}(t)\right) .\right.
$$

The time delays $h_{i j}($.$) are continuous and satisfy the following condition:$

$$
0 \leq h_{1} \leq h_{i j}(t)<h_{2}, \quad t \geq 0, \quad \forall i, j=\overline{1, N},
$$

and the initial function $\varphi(t)=\left[\varphi_{1}(t), \ldots \varphi_{N}(t)^{T}\right], \varphi_{i}(t) \in C^{1}\left(\left[-h_{2}, 0\right], R^{n_{i}}\right)$, with the norm

$$
\left\|\varphi_{i}\right\|=\sup _{-h \leq t \leq 0}\left\{\left\|\varphi_{i}(t)\right\|,\left\|\dot{\varphi}_{i}(t)\right\|\right\}, \quad\|\varphi\|=\sqrt{\sum_{i=1}^{N}\left\|\varphi_{i}\right\|^{2}} .
$$


Definition 1. Given $\alpha>0$. The zero solution of system (1) is $\alpha$-exponentially stable if there exist a positive number $N>0$ such that every solution $x(t, \varphi)$ satisfies the following condition:

$$
\|x(t, \varphi)\| \leq N e^{-\alpha t}\|\varphi\|, \quad \forall t \in R^{+} .
$$

Proposition 1. For any $x, y \in R^{n}$ and positive definite matrix $P \in R^{n \times n}$, we have

$$
2 x^{T} y \leq y^{T} P y+x^{T} P^{-1} x .
$$

Proposition 2. (Schur complement lemma [30]). Given constant matrices $X, Y, Z$ with appropriate dimensions satisfying $X=X^{T}, Y=Y^{T}>0$. Then $X+Z^{T} Y^{-1} Z<0$ if and only if

$$
\left(\begin{array}{cc}
X & Z^{T} \\
Z & -Y
\end{array}\right)<0 \quad \text { or } \quad\left(\begin{array}{cc}
-Y & Z \\
Z^{T} & X
\end{array}\right)<0
$$

Proposition 3. [32] For any constant matrix $Z=Z^{T}>0$ and scalar $h, \bar{h}, 0<h<\bar{h}$ such that the following integrations are well defined, then

$$
\begin{aligned}
&-\int_{t-h}^{t} x(s)^{T} Z x(s) d s \leq-\frac{1}{h}\left(\int_{t-h}^{t} x(s) d s\right)^{T} Z\left(\int_{t-h}^{t} x(s) d s\right) \\
&-\int_{-\bar{h}}^{-h} \int_{t+\theta}^{t} x(s)^{T} Z x(s) d s d \theta \leq-\frac{2}{\bar{h}^{2}-h^{2}}\left(\int_{-\bar{h}}^{-h} \int_{t+\theta}^{t} x(s) d s d \theta\right)^{T} \\
& Z\left(\int_{-\bar{h}}^{-h} \int_{t+\theta}^{t} x(s) d s d \theta\right) .
\end{aligned}
$$

\section{Main Results}

In this section, we investigate the decentralized exponential stability of nonlinear large-scale control system (1) with interval time-varying delays. It will be seen from the following theorem that neither free-weighting matrices nor any transformation are employed in our derivation. Before introducing main result, the following notations of several matrix variables are defined for simplicity.

$$
M_{11}^{i}=A_{i}^{T} P_{i}+A_{i} P_{i}+2 \alpha P_{i}+2 Q_{i}-2 S_{i 1} A_{i}+\sum_{j \neq i, j=1}^{N} P_{i} D_{i j} D_{i j}^{T} P_{i}
$$




$$
\begin{aligned}
& +\sum_{j \neq i, j=1}^{N} S_{i 1} D_{i j} D_{i j}^{T} S_{i 1}^{T}+S_{i 4} A_{i} A_{i}^{T} S_{i 4}^{T}+2 \beta_{i 1} P_{i}, \\
& M_{1 k}^{i}=-S_{i 4} A_{i}, \quad \forall k=\overline{2, N}, M_{1(N+1)}^{i}=-S_{i 2} A_{i}, M_{1(N+2)}^{i}=S_{i 3} A_{i}, \\
& M_{1(N+3)}^{i}=S_{i 1}-S_{i 5} A_{i}, M_{k k}^{i}=\sum_{j \neq i, j=1}^{N} S_{i 4} D_{i j} D_{i j}^{T} S_{i 4}^{T}+7 I+2 \beta_{i 2} P_{i}, \forall k=\overline{2, N}, \\
& M_{k(N+1)}^{i}=0, M_{k(N+2)}^{i}=0, M_{k(N+3)}^{i}=S_{i 4}, \\
& M_{(N+1)(N+1)}^{i}=-e^{2 \alpha h_{1}} Q_{i}+\sum_{j \neq i, j=1}^{N} S_{i 2} D_{i j} D_{i j}^{T} S_{i 2}^{T}, M_{(N+1)(N+2)}^{i}=0, \\
& M_{(N+1)(N+3)}^{i}=S_{i 2}, M_{(N+2)(N+2)}^{i}=-e^{2 \alpha h_{2}} Q_{i}+\sum_{j \neq i, j=1}^{N} S_{i 3} D_{i j} D_{i j}^{T} S_{i 3}^{T}, \\
& M_{(N+2)(N+3)}^{i}=S_{i 3}, M_{(N+3)(N+3)}^{i}=2 S_{i 5}+\sum_{j \neq i, j=1}^{N} S_{i 5} D_{i j} D_{i j}^{T} S_{i 5}^{T}, \\
& \lambda_{i 1}=\lambda_{\min }\left(P_{i}\right), \lambda_{1}=\min _{i=\overline{1, N}} \lambda_{i 1}, \lambda_{2}=\max _{i=\overline{1, N}} \lambda_{i 2}, \lambda_{i 2}=\lambda_{\max }\left(P_{i}\right)+\alpha^{-1} \lambda_{\max }\left(Q_{i}\right) .
\end{aligned}
$$

The following is the main result of the paper, which gives sufficient conditions for the decentralized exponential stability of nonlinear large-scale system (1) with interval time-varying delays. Essentially, the proof is based on the construction of Lyapunov Krasovskii functions satisfying Lyapunov stability theorem for time-delay system [30].

Theorem 1. Given $\alpha>0$. System (1) is $\alpha$-exponentially stable if there exist symmetric positive definite matrices $P_{i}, Q_{i}, i=\overline{1, N}$, and matrices $S_{i j}, i=$ $\overline{1, N}, j=1,2, \ldots, 5$ such that the following $L M I$ holds

$$
\mathcal{M}^{i}=\left[\begin{array}{cccc}
M_{11}^{i} & M_{12}^{i} & \ldots & M_{1(N+3)}^{i} \\
* & M_{22}^{i} & \ldots & M_{2(N+3)}^{i} \\
\cdot & \cdot & \ldots & \cdot \\
* & * & \ldots & M_{(N+3)(N+3)}^{i} \cdot
\end{array}\right]<0, \quad i=\overline{1, N}
$$

Moreover, the solution $x(t, \varphi)$ of the system satisfies

$$
\|x(t, \varphi)\| \leq \sqrt{\frac{\lambda_{2}}{\lambda_{1}}} e^{-\alpha t}\|\varphi\|, \quad \forall t \in R^{+} .
$$

Proof. We consider the following Lyapunov-Krasovskii functional for the system 
(1)

$$
V\left(t, x_{t}\right)=\sum_{i=1}^{N} \sum_{j=1}^{3} V_{i j}\left(t, x_{t}\right)
$$

where

$$
\begin{aligned}
V_{i 1} & =x_{i}^{T}(t) P_{i} x_{i}(t), \\
V_{i 2} & =\int_{t-h_{1}}^{t} e^{2 \alpha(s-t)} x_{i}^{T}(s) Q_{i} x_{i}(s) d s, \\
V_{i 3} & =\int_{t-h_{2}}^{t} e^{2 \alpha(s-t)} x_{i}^{T}(s) Q_{i} x_{i}(s) d s .
\end{aligned}
$$

It easy to verify that

$$
\sum_{i=1}^{N} \lambda_{i 1}\left\|x_{i}(t)\right\|^{2} \leq V\left(t, x_{t}\right), \quad V\left(0, x_{0}\right) \leq \sum_{i=1}^{N} \lambda_{i 2}\left\|\varphi_{i}\right\|^{2} .
$$

Taking the derivative of $V$ in $t$ along the solution of system (1), we have

$$
\begin{aligned}
\dot{V}_{i 1} & =2 x_{i}^{T}(t) P_{i} \dot{x}_{i}(t)=2 x_{i}^{T}(t)\left[A_{i}^{T} P_{i}+A_{i} P_{i}\right] x_{i}(t)+2 x_{i}^{T}(t) P_{i} D_{i j} x_{j}\left(t-h_{i j}(t)\right) \\
& +2 x_{i}^{T}(t) P_{i} \sum_{j \neq i, j=1}^{N} f_{i}\left(t, x_{i}(t), x_{j}\left(t-h_{i j}(t)\right) ;\right. \\
\dot{V}_{i 2} & =x_{i}^{T}(t) Q_{i} x_{i}(t)-e^{-2 \alpha h_{1}} x_{i}^{T}\left(t-h_{1}\right) Q_{i} x_{i}\left(t-h_{1}\right)-2 \alpha V_{i 2} ; \\
\dot{V}_{i 3} & =x_{i}^{T}(t) Q_{i} x_{i}(t)-e^{-2 \alpha h_{2}} x_{i}^{T}\left(t-h_{2}\right) Q_{i} x_{i}\left(t-h_{2}\right)-2 \alpha V_{i 3} .
\end{aligned}
$$

Note that

$$
\begin{aligned}
& \sum_{i=1}^{N} \sum_{j=1, j \neq i}^{N} x_{j}\left(t-h_{i j}(t)\right)^{T} x_{j}\left(t-h_{i j}(t)\right) \\
& =\sum_{i=1}^{N}\left[\sum_{j=1, i \neq j}^{N} x_{i}\left(t-h_{j i}(t)\right)^{T} x_{i}\left(t-h_{j i}(t)\right)\right] \\
& \sum_{i=1}^{N} \sum_{j=1, j \neq i}^{N} a_{i j} x_{j}\left(t-h_{i j}(t)\right)^{T} x_{j}\left(t-h_{i j}(t)\right)
\end{aligned}
$$




$$
=\sum_{i=1}^{N}\left[\sum_{j=1, i \neq j}^{N} a_{j i} x_{i}\left(t-h_{j i}(t)\right)^{T} x_{i}\left(t-h_{j i}(t)\right)\right\rfloor
$$

Therefore, we have

$$
\begin{aligned}
& \dot{V}(.)+2 \alpha V(.) \\
& \leq \sum_{i=1}^{N}\left[x _ { i } ^ { T } ( t ) \left[A_{i}^{T} P_{i}+A_{i} P_{i}+2 \alpha P_{i}+2 Q_{i}-2 S_{i 1} A_{i}\right.\right. \\
& \left.+\sum_{j \neq i, j=1}^{N} P_{i} D_{i j} D_{i j}^{T} P_{i}+\sum_{j \neq i, j=1}^{N} S_{i 1} D_{i j} D_{i j}^{T} S_{i 1}^{T}+S_{i 4} A_{i} A_{i}^{T} S_{i 4}^{T}+2 \beta_{i 1} P_{i}\right] x_{i}(t) \\
& +x_{i}^{T}\left(t-h_{1}\right)\left[-e^{2 \alpha h_{1}} Q_{i}+\sum_{j \neq i, j=1}^{N} S_{i 2} D_{i j} D_{i j}^{T} S_{i 2}^{T}\right] x_{i}\left(t-h_{1}\right) \\
& +x_{i}^{T}\left(t-h_{2}\right)\left[-e^{2 \alpha h_{2}} Q_{i}+\sum_{j \neq i, j=1}^{N} S_{i 3} D_{i j} D_{i j}^{T} S_{i 3}^{T}\right] x_{i}\left(t-h_{2}\right) \\
& +\dot{x}_{i}^{T}(t)\left[2 S_{i 5}+\sum_{j \neq i, j=1}^{N} S_{i 5} D_{i j} D_{i j}^{T} S_{i 5}^{T}\right] \dot{x}_{i}(t) \\
& +\sum_{j \neq i, j=1}^{N} x_{i}^{T}\left(t-h_{j i}(t)\right)\left[\sum_{j \neq i, j=1}^{N} S_{i 4} D_{i j} D_{i j}^{T} S_{i 4}^{T}+7 I+2 \beta_{i 2} P_{i}\right] x_{i}\left(t-h_{j i}(t)\right) \\
& \left.+2 x_{i}^{T}(t)\left[-S_{i 2} A_{i}\right] x_{i}\left(t-h_{1}\right)\right]+\sum_{i=1}^{N}\left[2 x_{i}^{T}(t)\left[S_{i 3} A_{i}\right] x_{i}\left(t-h_{2}\right)\right. \\
& +2 x_{i}^{T}(t)\left[S_{i 1}-S_{i 5} A_{i}\right] \dot{x}_{i}(t)+2 x_{i}^{T}\left(t-h_{1}\right) S_{i 2} \dot{x}_{i}(t)+2 x_{i}^{T}\left(t-h_{1}\right) S_{i 3} \dot{x}_{i}(t) \\
& +2 \sum_{j \neq i, j=1}^{N} x_{i}^{T}\left(t-h_{j i}(t)\right) S_{i 4} \dot{x}_{i}(t)-2 \sum_{j \neq i, j=1}^{N} x_{i}^{T}\left(t-h_{j i}(t)\right) S_{i 4} A_{i} x_{i}(t) \\
& =\sum_{i=1}^{N} \zeta_{i}^{T}(t) \mathcal{M}^{i} \zeta_{i}(t)
\end{aligned}
$$

where $\zeta_{i}^{T}(t)=\left[x_{i}^{T}(t), x_{i}^{T}\left(t-h_{1}\right), x_{i}^{T}\left(t-h_{2}\right),\left(x_{i}^{T}\left(t-h_{j i}\right)\right)_{j \neq i, j=1}^{N}, \dot{x}_{i}^{T}(t)\right]$.

By condition (2), we obtain

$$
\dot{V}\left(t, x_{t}\right) \leq-2 \alpha V\left(t, x_{t}\right), \quad \forall t \in R^{+}
$$


Integrating both sides of (4) from 0 to $t$, we obtain

$$
V\left(t, x_{t}\right) \leq V(\varphi) e^{-2 \alpha t}, \quad \forall t \in R^{+} .
$$

Furthermore, taking condition (3) into account, we have

$$
\lambda_{1}\|x(t, \varphi)\|^{2} \leq V\left(x_{t}\right) \leq V(\varphi) e^{-2 \alpha t} \leq \lambda_{2} e^{-2 \alpha t}\|\varphi\|^{2},
$$

then

$$
\|x(t, \varphi)\| \leq \sqrt{\frac{\lambda_{2}}{\lambda_{1}}} e^{-\alpha t}\|\varphi\|, \quad t \in R^{+} .
$$

This completes the proof of the theorem.

Remark 2. Theorem 1 provides sufficient conditions for nonlinear largescale system (1) in terms of the solutions of LMIs, which guarantees the closedloop system to be exponentially stable with a prescribed decay rate $\alpha$. The developed method using new inequalities for lower bounding cross terms eliminate the need for over bounding and provide larger values of the admissible delay bound. Note that the time-varying delays are non-differentiable, therefore, the methods proposed in [1-31] are not applicable to system (1). The LMI condition (2) depends on parameters of the system under consideration as well as the delay bounds. The feasibility of the LMIs can be tested by the reliable and efficient Matlab LMI Control Toolbox [30].

\section{Conclusion}

In this paper, the problem of the decentralized exponential stability for nonlinear large-scale time-varying delay systems has been studied. The time delay is assumed to be a function belonging to a given interval, but not necessary to be differentiable. The developed method using new inequalities for lower bounding cross terms eliminate the need for over-bounding and provide larger values of the delay bound.

\section{Acknowledgments}

This work was supported by the Thailand Research Fund Grant, the Commission for Higher Education and Faculty of Science, Maejo University, Thailand. The authors thank anonymous reviewers for valuable comments and suggestions, which allowed us to improve the paper. 


\section{References}

[1] M.S. Mahmoud, Improved Stability and stabilization approach to linear interconnected time-delay systems, Optim. Control Appl. Methods 31 (2010), 81-92.

[2] M.S. Mahmoud, Decentralized reliable control of interconnected systems with time-varying delays, J. Optim. Theory Appl. 143 (2009), 497-518.

[3] Manlika Ratchagit, On stability of switched linear systems, International Journal of Pure and Applied Mathematics, Vol. 78 No. 6, 2012, 849-856.

[4] K. Ratchagit, Asymptotic stability of delay-difference system of Hopfield neural networks via matrix inequalities and application, International Journal of Neural Systems, 17 (2007), 425-430. DOI: 10.1142/S0129065707001263

[5] S.G. Wang, H.S. Yao, Pinning synchronization of the time-varying delay coupled complex networks with time-varying delayed dynamical nodes, Chin.Phys. B 21 (2012), 050508-1-050508-2.

[6] S. Wang, H. Yao, S. Zheng, Y. Xie, A novel criterion for cluster synchronization of complex dynamical networks with coupling time-varying delays, Commun. Nonlinear Sci. Numer. Simul. 17 (2012), 2997-3004.

[7] Kreangkri Ratchagit, Stability criteria of lpd system with time-varying delay, International Journal of Pure and Applied Mathematics, Vol. 78 No. 6, 2012, 857-866.

[8] Kreangkri Ratchagit, Stability analysis of linear systems with time delays, International Journal of Pure and Applied Mathematics, Vol. 76 No. 1, 2012, 21-28.

[9] Kreangkri Ratchagit, Stability of linear time-varying systems, International Journal of Pure and Applied Mathematics, Vol. 63 No. 4, 2010, 411-417.

[10] Kreangkri Ratchagit, Exponential stability of switched linear systems, International Journal of Pure and Applied Mathematics, Vol. 58 No. 3, 2010, 361-371.

[11] K. Ratchagit, The sufficient conditions for stability of linear time-varying systems with state delays, International Journal of Pure and Applied Mathematics, Vol. 65 No. 1, 2010, 65-72. 
[12] G. Rajchakit, Stabilization of switched discrete-time systems with convex polytopic uncertainties, Journal of Computational Analysis and Applications 16 (2014), 20-29.

[13] K. Ratchagit and V.N. Phat, Stability criterion for discrete-time systems, J. Ineq. Appl., 2010 (2010), 1-6. doi:10.1155/2010/201459

[14] G. Rajchakit, Switching design for the robust stability of nonlinear uncertain stochastic switched discrete-time systems with interval time-varying delay. Journal of Computational Analysis \& Applications 16 (2014), 10-19.

[15] M. De la Sen and A. Ibeas, Stability Results of a Class of Hybrid Systems under Switched Continuous-Time and Discrete-Time Control, Discrete Dynamics in Nature and Society, vol. 2009, Article ID 315713, 28 pages, 2009. doi:10.1155/2009/315713

[16] G. Rajchakit, Robust stability and stabilization of nonlinear uncertain stochastic switched discrete-time systems with interval time-varying delays. Applied Mathematics and Information Sciences 6 (2012), 555-565.

[17] M. de la Sen and A. Ibeas, Stability Results for Switched Linear Systems with Constant Discrete Delays, Mathematical Problems in Engineering, vol. 2008, Article ID 543145, 28 pages, 2008. doi:10.1155/2008/543145

[18] G. Rajchakit, Delay-dependent optimal guaranteed cost control of stochastic neural networks with interval nondifferentiable time-varying delays, Advances in Difference Equations, 2013 (2013), 1-11. DOI: 10.1186/16871847-2013-241

[19] K. Ratchagit, A switching rule for the asymptotic stability of discrete-time systems with convex polytopic uncertainties, Asian-European J. Math., 5 (2012), 1250025 (12 pages),. DOI: 10.1142/S1793557112500258

[20] G. Rajchakit, Stabilization of switched discrete-time systems with convex polytopic uncertainties, Journal of Computational Analysis \& Applications 16 (2014), 20-29.

[21] Manlika Rajchakit, Piyapong Niamsup, Grienggrai Rajchakit, A constructive way to design a switching rule and switching regions to mean square exponential stability of switched stochastic systems with non-differentiable and interval time-varying delay, Journal of Inequalities and Applications 2013, 2013:499 doi:10.1186/1029-242X-2013-499 
[22] Grienggrai Rajchakit, Delay-Dependent Asymptotical Stabilization Criterion of Recurrent Neural Networks, Applied Mechanics and Materials. 330 (2013), 1045-1048. doi:10.4028/www.scientific.net/AMM.330.1045

[23] K. Ratchagit, Asymptotic stability of nonlinear delay-difference system via matrix inequalities and application, International Journal of Computational Methods, pp. 389-397, 2009. DOI: 10.1142/S0219876209001899

[24] M. de la Sen, Global Stability of Polytopic Linear Time-Varying Dynamic Systems under Time-Varying Point Delays and Impulsive Controls, Mathematical Problems in Engineering, vol. 2010, Article ID 693958, 33 pages, 2010. doi: $10.1155 / 2010 / 693958$

[25] G. Rajchakit, Exponential stability of switched linear systems with interval time-varying delays, Proceedings of the 2012 IEEE International Conference on Robotics and Biomimetics December 11-14, 2012, Guangzhou, China, 1502-1506. doi: 10.1109/ROBIO.2012.6491181

[26] K. Ratchagit, V.N. Phat, Stability and stabilization of switched linear discrete-time systems with interval time-varying delay, Nonlinear Anal. Hybrid Syst. 5 (2011), 605-612. DOI: 10.1016/j.nahs.2011.05.006

[27] M. De la Sen, On the Characterization of Hankel and Toeplitz Operators Describing Switched Linear Dynamic Systems with Point Delays, Abstract and Applied Analysis, vol. 2009, Article ID 670314, 34 pages, 2009. doi:10.1155/2009/670314

[28] VN. Phat, Y. Kongtham, and K. Ratchagit, LMI approach to exponential stability of linear systems with interval time-varying delays, Linear Algebra Appl., Vol. 436, pp. 243-251, 2012. doi: 10.1016/j.laa.2011.07.016

[29] P. Niamsup, M. Rajchakit, G. Rajchakit, Guaranteed cost control for switched recurrent neural networks with interval time-varying delay, JOURNAL OF INEQUALITIES AND APPLICATIONS, 2013 (2013),. DOI: $10.1186 / 1029-242 \mathrm{X}-2013-292$

[30] P. Niamsup, G. Rajchakit, New Results on Robust Stability and Stabilization of Linear Discrete-Time Stochastic Systems with Convex Polytopic Uncertainties, JOURNAL OF APPLIED MATHEMATICS, 2013 (2013),. DOI: $10.1155 / 2013 / 368259$ 
[31] G. Rajchakit, Stabilization of switched discrete-time systems with convex polytopic uncertainties, Journal of Computational Analysis and Applications 16 (2014), 20-29.

[32] R.P. Agarwal, Difference Equations and Inequalities, Second Edition, Marcel Dekker, New York, 2000. 